\title{
Reinforcement Learning
}

\author{
Merin Deora $^{1}$, Sumit Mathur ${ }^{2}$ \\ Student, Computer Science Department, Poornima Group of Institutions, Jaipur, Rajasthan, India ${ }^{1}$ \\ Asst. Professor, Computer Science Department, Poornima Group of Institutions, Jaipur, Rajasthan, India ${ }^{2}$
}

\begin{abstract}
Support learning (RL) is accepted to be relate fitting worldview for deed administration approaches in versatile manmade brainpower. Be that as it may, in its ordinary definition (clean slate) RL ought to investigate and take in everything starting with no outside help that is neither reasonable nor viable in true undertakings. In this article we tend to propose a substitution system, known as administered Reinforcement Learning (SRL), for exploiting outside data inside this sort of learning and approve it in an exceedingly divider taking after conduct. Mechanical autonomy is one among the preeminent troublesome uses of Machine Learning (ML) systems. It's portrayed by direct collaboration with a genuine world, tactile criticism and an enormous many-sided quality of the framework. As of late many ways to deal with utilize mil to specie counterfeit consciousness undertakings are uncovered. Despite we square measure still far away from a whole self-sufficient robot framework with learning parts.
\end{abstract}

Keywords: Machine Learning; Brute force Algorithm.

\section{INTRODUCTION}

Support learning (RL)is a noteworthy technique for the determination of assignments in various areas, similar to diversion playing (Tesauro, 1994), mechanical technology (Schaal and Atkeson, 1994) and even portable workstation systems (Boyan and Littman, 1994). One among the most points of interest of RL is that it doesn't might want a gathering of contributions with their right responses for honing that zone unit regularly difficult to give in dynamic and obscure situations. Rather, it exclusively needs a measure of the framework's level of conduct, assumed fortification. This component and its dynamic nature and accommodating capacities make is fitting to be utilized as a part of portable AI.

These restrictions cause absence of dependability all through adapting, low levels of strength inside the learnt practices, and gradualness in joining. These issues turn out to be much more clear in genuine applications, especially in portable apply autonomy (Wyatt, 1995), wherever conditions region unit confounded, dynamic, and not normally entirely show capable .However, in these frameworks there regularly exists past data on the errand inside the kind of human experience or previously created controllers. This data might be utilized to enhance the preparation strategy, on the grounds that the RL operator does not start sans preparation.

Huge numbers of the frameworks refered to on top of impact exclusively horribly particular AI issues or with simplications that make the progression from a mimicked to a genuine surroundings exceptionally troublesome. Frequently in view of the undeniable reality that at the moment ML-systems and AI issues don't coordinate well. A few thoughts in machine learning territory unit connected to very basic "universes" exclusively.

The application we tend to utilize in order to check our framework engineering is that the assignment of figuring out how to know and to attempt to simple controls with from the earlier obscure protests via consequently playing tests by the framework and by abuse techniques that are removed from client cases replicated all through a client assurance these or comparable errands.

\section{REINFORCEMENT LEARNING}

In the RL worldview (Kaelbling et al., 1996), an operator cooperates with the setting through an accumulation of activities. The setting is then changed and in this way the operator sees the new state through its sensors. Besides, at each progression the operator gets an outer reward flag the objective of the RL specialist is to expand the measure of reward got inside the long run.

In machine taking in, the earth is ordinarily figured as a Markov decision prepare (MDP) a similar number of bolster learning estimations for this setting use dynamic programming techniques.[1] The essential differentiation between the built up frameworks and fortress learning figuring's is that the last needn't trouble with data about the MDP and they target tremendous MDPs where revise methodologies get the opportunity to be unmistakably infeasible.

In this learning procedure a goal is illustrated and the learning technique happens through experimentation connections amid a dynamic setting. The operator is remunerated on censured on the introduce of the activities it does. There square measure a few calculations that actualize RL standards, among the chief utilized square measure Sarsa, Dyna, 
Prioritized Sweeping and Q-Learning. Amid this work we tend to have used the last one (Watkins, 1989) as, because of its straightforwardness and basic usage, is that the one that is directly the first regularly utilized.

Learning should bring about snappier drawback arrangements, quicker or extra dependable answer executions or to the adaptability to fathom issues the system wasn't prepared to understand some time recently. The investigation of today's instrument administration frameworks and of Run of the mill AI issues on one aspect and subsequently the investigation of ML-methods on the inverse feature demonstrated an outsized hole between these 2 examination territories. Table one gives AN outline of the preeminent important varieties.

Support taking in contrasts from standard regulated learning in that right info/yield sets are never exhibited, nor problematic activities unequivocally redressed. Encourage, there is an attention on-line execution, which includes finding a harmony between investigation (of unfamiliar domain) and misuse (of current knowledge).[2] The investigation versus misuse exchange off in fortification learning has been most altogether concentrated through the multi-furnished criminal issue and in limited MDPs.

\section{Algorithms for control learning:-}

Criterion of optimality:-For effortlessness, accept for a minute that the issue examined is verbose, a scene consummation when some terminal state is come to. Accept facilitate that regardless of what course of moves the operator makes, end is inescapable. Under some mellow consistency conditions the desire of the aggregate reward is then all around characterized, for any arrangement and any underlying conveyance over the states. Here, a strategy alludes to a mapping that allocates some likelihood dissemination over the activities to all conceivable histories.

Given a settled introductory conveyance \{\displaystyle $\backslash \mathrm{mu}\} \backslash \mathrm{mu}$, we can subsequently dole out the normal return $\left\{\right.$ Idisplaystyle $\backslash$ rho $\left.\wedge^{\wedge}\{\mid \mathrm{pi}\}\right\} \backslash \mathrm{rho} \wedge\{$ |pi $\}$ to approach $\{$ Idisplaystyle $\mid \mathrm{pi}\} \backslash \mathrm{pi}$ :

$$
\rho^{\pi}=E[R \mid \pi],
$$

Where the random variable denotes the return and is defined by

$$
R=\sum_{t=0}^{N-1} r_{t+1},
$$

Where $\left\{\right.$ ddisplay style $\left.r_{-}\{t+1\}\right\} r_{-}\{t+1\}$ is the reward got after the $\{$ \display style $t\} t$-th move, the underlying state is tested aimlessly from \{ \display style $\backslash \mathrm{mu}\} \backslash \mathrm{mu}$ and activities are chosen by arrangement $\{$ \display style $\backslash \mathrm{pi}\} \backslash \mathrm{pi}$. Here, \{ldisplay style $\mathrm{N}$ \} $\mathrm{N}$ indicates the (arbitrary) time when a terminal state is come to, i.e., the time when the scene ends.

$$
R=\sum_{t=0}^{\infty} \gamma^{t} r_{t+1},
$$

Offering ascend to the aggregate expected marked down reward measure. Here \{ldisplaystyle 0lleq Igamma leq 1\} 0lleq Igamma leq 1 is the purported rebate consider. Since the undiscounted return is a unique instance of the reduced return, starting now and into the foreseeable future we will expect marking down. Despite the fact that this looks sufficiently guiltless, marking down is in truth risky in the event that one thinks about online execution. This is on account of marking down makes the underlying time steps more essential. Since a learning operator is probably going to commit errors amid the initial few stages after its "life" begins, no ignorant learning calculation can accomplish close ideal execution under marking down regardless of the possibility that the class of conditions is confined to that of limited MDPs. (This does not mean however that, sufficiently given time, a learning operator can't figure the proper behavior close ideally, if time was restarted.)

The issue then is to determine a calculation that can be utilized to discover an arrangement with most extreme expected return. From the hypothesis of MDPs it is realized that, without loss of all-inclusive statement, the pursuit can be limited to the arrangement of the supposed stationary approaches. An arrangement is called stationary if the activity appropriation returned by it depends just on the last state went by (which is a piece of the perception history of the specialist, by our improving presumption). Truth be told, the pursuit can be further limited to deterministic stationary approaches [3]. A deterministic stationary approach is one which deterministically chooses activities in view of the present state. Since any such arrangement can be related to a mapping from the arrangement of states to the arrangement of activities, these approaches can be related to such mappings with no loss of sweeping statement.

Brute force :- The savage compel approach involves the accompanying two stages:

(1) For every conceivable approach, test returns while tailing it Pick the strategy with the biggest expected return One issue with this is the quantity of strategies can be to a great degree vast, or even interminable. Another is that change of the profits may be vast, in which case an expansive number of tests will be required to precisely appraise the arrival of every strategy. 
(2) These issues can be enhanced in the event that we accept some structure and maybe permit tests produced from one approach to impact the appraisals made for another. The two primary methodologies for accomplishing this are esteem work estimation and direct strategy look.

\section{APPLICATION}

The administration of portable robots is to a great degree progressed, and needs the work of administration structures (Regueiro et al., 2002). The heft of those are construct for the most part with respect to low-level practices that speedily resolve a specific Undertaking. We have picked the divider taking after conduct as the undertaking to be learnt, in light of the fact that it is one in all the first used in portable counterfeit consciousness. RL Agent: The 3 parts of a RL framework are: the state illustration, the compensate work and furthermore the activities that the golem will retain each state [4]. At the point when many coming up short investigations, we have a tendency to needed to plot a fifth state variable: the relative introduction $(\mathrm{O})$ between the golem and furthermore the divider. To get this the reference divider. Is approximated to a line by implies that of straight relapse of the latest estimations from sensors eleven, 12 and thirteen (Figure 3(a)). The point between this straight line and furthermore the forward heading of the golem is discretized into four qualities: drawing nearer, moving endlessly, parallel and no heading. The last cost is dispensed once the relapse is not dependable, the frontal divider is fantastically closed, or the golem is to stop to or too from the divider being taken after.

Earlier data: - In our framework we have utilized one PKS, to make it we have a tendency to have utilized an identity's expert to go to a choice on the activity to be dispensed in an exceedingly scope of agent positions Inside the climate. This PKS (called Ad-Hoc) gives sensibly savvy proposal in twelve states relating to the straight divider and open corner things. For each State with proposal exclusively Associate in nursing activity with most utility is directed. It's indispensable to stress that all alone, the specialist isn't able to do completely breakdown the undertaking.

\section{EXPERIMENTAL RESULTS}

Trial strategy: - In our tests we tend to separate between administration cycles and learning steps. The past are apportioned every $1 / 3$ second, and here the framework refreshes the sensor, Measurements, decides the present state, and chooses the activity to be implemented. Still, the instructive Steps exclusively occur with the operator changes state, because of that they're nonconcurrent.

Joining time: - It demonstrates the outcomes for the RL specialist (Q-learning). This figure demonstrates the reward got all through the instructive stage: each reason speaks to the fortification amassed in the past one, 000 learning cycles. It can be seen however the instructive technique balances out at around nineteen, 000 learning steps. This figure conjointly demonstrates the aftereffects of the test part (control cycles while not disappointment).

Strength of the learnt conduct: - a definitive point of the instructive technique is for the machine to achieve figuring out how to take after the reference divider. Be that as it may, it is conjointly essential to confirm just however solid a definitive Learnt conduct is, and a method for approving this is regularly to study what happens once the setting changes. On the off chance that it is powerful, it'll not be influenced to any pleasant degree, and the machine will be equipped for twisting up the undertaking.

\section{RELATED WORK}

Endeavors designed for together with specialists' past data into RL inside the field of versatile manmade brainpower are bolstered three methods. The essential is that the style of muddled fortifications (Mataric', 1994), that has the matter of its absence of all-inclusive statement, and furthermore the issue brought about by generation the capacities that offer the variable support, which will without a doubt not be made utilization of in the other errand. Dynamic learning orientated making of the state space (Hailu, 2001) is that the second procedure. Its principle downsides square measure that the soundness of the framework all through learning isn't thought about, which there's a Great reliance on the standard of information: if this can be off base, the specialist can't learn. The third and most imperative approach is that the centralization of investigation. There square measure various methodologies, one Being to center investigation exclusively at the onset of learning (Del R. Mill'an et al., 2002). The connected math nature of RL winds up in there being variances inside the learning procedure, which can bring about the downgrading of partner degree Initiallysuggested sensible activity. In these cases, the underlying centralization of the investigation doesn't encourage to balance out the merging of the RL run the show. 


\section{CONCLUSION AND FUTURE WORK}

The utilization of fortification learning (RL) in genuine frameworks has highlighted the limitations of those calculations, the fundamental one being gradualness in joining. On the inverse hand, in genuine frameworks there as a rule exists past data on the undertaking being learnt that might be wont to enhance the learning technique. In this paper we have a tendency to propose a trade methodology for making utilization of outer data at interims RL that we tend to choice directed Reinforcement Learning (SRL). The SRL is predicated on abuse past data on the undertaking to center the RL calculation's investigation towards the chief promising territories of the state range. Due to SRL, data are regularly used to accelerate joining of RL calculations, yielding at indistinguishable time extra solid controllers and up the operator's steadiness all through the instructive technique. So as to show the feasibility of the anticipated Methodology it's been connected to the determination of an essential errand in portable manmade brainpower, the divider taking after conduct, and it's been contrasted and an established clean slate RL run (Q-learning). At the moment we tend to range unit following up on the usage of the spoke to thought. Our testbed comprises of a painter 260B with constrain torque-sensor, hand camera, Unival administration, vision equipment and Sun workstations with Common Lisp for the mental element framework[5].

We apply our idea to the procurement of protest learning then the getting a handle on and control of these items from possess tests and by client guiding. These assignments kind the thought for the apparatus of Recycling (dismantling) of (mostly) obscure items we wish to handle in later periods of the venture. Future work likewise will be devoted to the blend of responsive sub typical ability parts bolstered connectionist. Approaches and furthermore the expansion of our 2Ddescription to 3D-primitives.

\section{REFERENCES}

[1] Using Prior Knowledge to Improve Reinforcement Learning in Mobile Robotics David L. Moreno, Carlos V. Regueiro†, Roberto Iglesias and Senen Barro.

[2] Application of machine learning to robotics \{an analysis Jürgen Kreuziger Institute for Real-Time Computer Control Systems \& Robotics Prof. Dr.-Ing. U.Rembold and Prof. Dr.-Ing. R.Dillmann.

[3] C.M. Kadie. Continuous conceptual set covering: Learning robot operators from examples. In Proc. 8th Int. Workshop on ML, Evanston, pp. $615\{619,1991$.

[4] R. Mill'an, J., Posenato, D., and Dedieu, E. (2002). Continuous-action q-learning. Machine Learning, 49:247, 265.

[5] Wyatt, J. (1995). Issues in putting reinforcement learning onto robots. In 10th Biennal Conference of the AISB, Sheffield, UK. 